\title{
Exact Solutions of Kuramoto-Sivashinsky Equation
}

\author{
Dahe Feng ${ }^{\mathrm{a}, \mathrm{b}}$
}

${ }^{a}$ School of Mathematical Science and Computing Technology, Central South University Changsha, China

${ }^{b}$ School of Mathematics and Computing Science Guilin University of Electronic Technology Guilin, China

\begin{abstract}
Many nonlinear partial differential equations admit traveling wave solutions that move at a constant speed without changing their shapes. It is very important and difficult to search the exact travelling wave solutions. In this work, the auxiliary Riccati equation method and the computer symbolic system Maple are used to study exact solutions for the nonlinear Kuramoto-Sivashinsky equation. Maple can help us solve tedious algebraic calculation. Therefore many exact traveling wave solutions are successfully obtained which include some new kink (or anti-kink) wave solutions and periodic wave solutions.
\end{abstract}

Index Terms: Kuramoto-Sivashinsky equation; auxiliary Riccati equation method; exact solution; kink wave solution; periodic wave solution

(C) 2012 Published by MECS Publisher. Selection and/or peer review under responsibility of the International Conference on E-Business System and Education Technology

\section{Introduction}

Nonlinear partial differential equations are widely used to describe complex phenomena in vary scientific fields and especially in areas of physics such as plasma, fluid mechanics, biology, solid state physics, nonlinear optics and so on. Therefore the investigation of the exact solutions to nonlinear equations plays an important role in the study of nonlinear science. Up to now, many powerful methods to seek for exact solutions of the nonlinear differential equations have been proposed. Among these are inverse scattering method [1], Lie group method [2], bifurcation method of dynamic systems [3,4], sine-cosine method [5], tanh function method [6], homogenous balance method [7]. [8]

Our interest in this work is to study the exact solutions of the following Kuramoto-Sivashinsky (KS) equation

$u_{t}+\alpha u u_{x}+\beta u_{x x}+\gamma u_{x x x x}=0$,

(1)

where $\alpha, \beta, \gamma$ are arbitrary constants. The KS equation (1) describes the fluctuations of the position of a flame front, the motion of a fluid going down a vertical wall. This equation was examined as a prototypical example

Corresponding author:

E-mail address: dahefeng@hotmail.com 
of spatiotemporal chaos in one space dimension [9]. Recently some new soliton solutions are obtained by the tanh method and the extended tanh method [8]. Here we use the auxiliary Riccati equation method to discuss the exact solutions of (1). With the aid of computer software Maple, we successfully obtain many new kink wave solutions and periodic wave solutions.

\section{Auxiliary Riccati Equation Method}

For a given partial differential equation with $u(x, t)$

$P\left(u, u_{t}, u_{x}, u_{t t}, u_{x t}, u_{x x}, \cdots\right)=0$,

can be converted to an ordinary differential equation

$P\left(u, u^{\prime}, u^{\prime \prime}, u^{\prime \prime \prime}, \cdots\right)=0$

under the traveling wave transformation $\xi=x-c t$.

Suppose that the solutions of (3) can be expressed in the form

$u=a_{0}+a_{1} \phi+a_{2} \phi^{2}+\cdots+a_{n} \phi^{n}$

with the new variable $\phi=\phi(\xi)$ satisfying the Riccati equation

$\phi^{\prime}=p+q \phi^{2}$,

where $\phi^{\prime}=(\mathrm{d} \phi) /(\mathrm{d} \xi), \xi=x-c t$ and $p, q$ are constants.

The Riccati equation (5) has special solutions as follows:

$$
\begin{aligned}
& p=1 / 2, q=-1 / 2, \phi_{1}=\tanh (\xi / 2), \operatorname{coth}(\xi / 2) ; \\
& p=1 / 2, q=1 / 2, \phi_{2}=\tan (\xi / 2),-\cot (\xi / 2) ; \\
& p=1, q=-1, \phi_{3}=\tanh \xi, \operatorname{coth} \xi ; \\
& p=1, q=1, \phi_{4}=\tan \xi,-\cot \xi ; \\
& p=1, q=-4, \phi_{5}=1 / 2 \tanh (2 \xi), 1 / 2 \operatorname{coth}(2 \xi) ; \\
& p=1, q=4, \phi_{6}=1 / 2 \tan (2 \xi),-1 / 2 \cot (2 \xi) .
\end{aligned}
$$

The process of solving (2) is taking as the following:

Firstly, we determine $n$ in (4) by substituting (4) and (5) into (3) and balancing the highest-order derivative terms with the highest-order nonlinear terms.

Secondly, substituting (5) and (4) with the concrete $n$ into (3) yields to an algebraic system of equations in powers of $\phi$. Then setting all coefficients of $\phi^{i}$ to be zero will lead to the determination of the parameters $a_{i}(i=0, \cdots, n)$.

Lastly, substituting all the obtained parameters $a_{i}$ into (4) and using special solutions of (5) gives the responding exact solutions of (2).

\section{Traveling Wave Solutions of The KS Equation}

This work is supported by the National Natural Science Foundation of China (11061010 and 61004101) and the Postdoctoral Science Foundation of Central South University. 
We then consider the KS equation (1). Making the traveling wave transformation $u(x, t)=u(\xi), \xi=x-c t$, then (1) reduces to the ordinary differential equation

$-c u^{\prime}+\alpha u u^{\prime}+\beta u^{\prime \prime}+\gamma u^{(4)}=0$.

Integrating (7) with respect to $\xi$ once yields

$-c u+\alpha / 2 u^{2}+\beta u^{\prime}+\gamma u^{\prime \prime \prime}+g=0$,

where $g$ is an integral constant. Substituting (4) and (5) into (8) and balancing $u^{\prime \prime \prime}$ with $u^{2}$ gives $n=3$.

Therefore it follows from (4) that we can choose the following ansatz:

$u(\xi)=a_{0}+a_{1} \phi+a_{2} \phi^{2}+a_{3} \phi^{3}$.

Substituting (9) into (8) with (5), collecting the coefficients of $\phi^{i}(i=0,1, \cdots, 6)$ and setting all them to zero, we obtain a system of algebraic equations. Solving the system by Maple gives the following solutions:

(i) The first set:

$a_{0}=\frac{c}{\alpha}, a_{1}=\frac{360 \gamma p q^{2}}{-\alpha}, a_{2}=0, a_{3}=\frac{120 \gamma q^{3}}{-\alpha}, \beta=76 \gamma p q$.

(ii) The second set:

$a_{0}=\frac{c}{\alpha}, a_{1}=\frac{1080 \gamma p q^{2}}{-11 \alpha}, a_{2}=0, a_{3}=\frac{120 \gamma q^{3}}{-\alpha}, \beta=\frac{76 \gamma p q}{-11}$.

This in turn gives the following general set of solutions

$u_{I}=\frac{c}{\alpha}-\frac{360 \gamma p q^{2}}{\alpha} \phi-\frac{120 \gamma q^{3}}{\alpha} \phi^{3}, \beta=76 \gamma p q$,

$u_{I I}=\frac{c}{\alpha}-\frac{1080 \gamma p q^{2}}{11 \alpha} \phi-\frac{120 \gamma q^{3}}{\alpha} \phi^{3}, \beta=-\frac{76 \gamma p q}{11}$,

where $p$ and $q$ are arbitrary constants and $\phi$ takes many trigonometric and hyperbolic functions as shown in (6) .

A. Exact solutions for case (12)

We first consider $u_{I}(x, t)$ by using the first set (10) and applying the related $\phi$ functions for this choice of $p$ and $q$.

Using the first case in (6) where $p=1 / 2, q=-1 / 2$ gives the hyperbolic function solution

$u_{1}(\xi)=\frac{c}{\alpha}-\frac{45 \gamma}{\alpha} \operatorname{coth} \frac{\xi}{2}+\frac{15 \gamma}{\alpha} \operatorname{coth}^{3} \frac{\xi}{2}$

and the kink wave solution

$u_{2}(\xi)=\frac{c}{\alpha}-\frac{45 \gamma}{\alpha} \tanh \frac{\xi}{2}+\frac{15 \gamma}{\alpha} \tanh ^{3} \frac{\xi}{2}$ 
where $\beta=-19 \gamma$.

For $p=1 / 2, q=1 / 2$, we find $\beta=19 \gamma$ and therefore obtain the two periodic traveling wave solutions

$u_{3}(\xi)=\frac{c}{\alpha}-\frac{45 \gamma}{\alpha} \tan \frac{\xi}{2}-\frac{15 \gamma}{\alpha} \tan ^{3} \frac{\xi}{2}$,

$u_{4}(\xi)=\frac{c}{\alpha}+\frac{45 \gamma}{\alpha} \cot \frac{\xi}{2}+\frac{15 \gamma}{\alpha} \cot ^{3} \frac{\xi}{2}$.

For $p=1, q=-1$, we gain $\beta=-76 \gamma$ and thus determine the hyperbolic function solution

$u_{5}(\xi)=\frac{c}{\alpha}-\frac{360 \gamma}{\alpha} \operatorname{coth} \xi+\frac{120 \gamma}{\alpha} \operatorname{coth}^{3} \xi$,

and the kink (or anti-kink) wave solution

$u_{6}(\xi)=\frac{c}{\alpha}-\frac{360 \gamma}{\alpha} \tanh \xi+\frac{120 \gamma}{\alpha} \tanh ^{3} \xi$.

For $p=1, q=1$, we find $\beta=76 \gamma$ and hence obtain the two periodic traveling wave solutions

$u_{7}(\xi)=\frac{c}{\alpha}-\frac{360 \gamma}{\alpha} \tan \xi-\frac{120 \gamma}{\alpha} \tan ^{3} \xi$,

$u_{8}(\xi)=\frac{c}{\alpha}+\frac{360 \gamma}{\alpha} \cot \xi+\frac{120 \gamma}{\alpha} \cot ^{3} \xi$.

For $p=1, q=-4$, we obtain $\beta=-304 \gamma$ and therefore give the hyperbolic function solution

$u_{9}(\xi)=\frac{c}{\alpha}-\frac{2880 \gamma}{\alpha} \operatorname{coth}(2 \xi)+\frac{960 \gamma}{\alpha} \operatorname{coth}^{3}(2 \xi)$,

and the kink (or anti-kink) wave solution

$u_{10}(\xi)=\frac{c}{\alpha}-\frac{2880 \gamma}{\alpha} \tanh (2 \xi)+\frac{960 \gamma}{\alpha} \tanh ^{3}(2 \xi)$.

For $p=1, q=4$, we find $\beta=304 \gamma$ and therefore obtain the two periodic traveling wave solutions

$u_{11}(\xi)=\frac{c}{\alpha}-\frac{2880 \gamma}{\alpha} \tan (2 \xi)-\frac{960 \gamma}{\alpha} \tan ^{3}(2 \xi)$,
$u_{12}(\xi)=\frac{c}{\alpha}+\frac{2880 \gamma}{\alpha} \cot (2 \xi)+\frac{960 \gamma}{\alpha} \cot ^{3}(2 \xi)$.

B. Exact solutions for case (13)

We next discuss $u_{I I}(x, t)$ by using the second set (11) and applying the related $\phi$ functions for this choice 
of $p$ and $q$.

Using the first case in (6) where $p=1 / 2, q=-1 / 2$ yields the hyperbolic function solution

$u_{13}(\xi)=\frac{c}{\alpha}-\frac{135 \gamma}{11 \alpha} \operatorname{coth} \frac{\xi}{2}+\frac{15 \gamma}{\alpha} \operatorname{coth}^{3} \frac{\xi}{2}$

and the kink wave solution

$u_{14}(\xi)=\frac{c}{\alpha}-\frac{135 \gamma}{11 \alpha} \tanh \frac{\xi}{2}+\frac{15 \gamma}{\alpha} \tanh ^{3} \frac{\xi}{2}$,

where $\beta=19 \gamma / 11$.

For $p=1 / 2, q=1 / 2$, we find $\beta=-19 \gamma / 11$ and therefore obtain the two periodic traveling wave solutions

$u_{15}(\xi)=\frac{c}{\alpha}-\frac{135 \gamma}{11 \alpha} \tan \frac{\xi}{2}-\frac{15 \gamma}{\alpha} \tan ^{3} \frac{\xi}{2}$,

$u_{16}(\xi)=\frac{c}{\alpha}+\frac{135 \gamma}{11 \alpha} \cot \frac{\xi}{2}+\frac{15 \gamma}{\alpha} \cot ^{3} \frac{\xi}{2}$.

For $p=1, q=-1$, we obtain $\beta=76 \gamma / 11$ and therefore give the hyperbolic function solution

$u_{17}(\xi)=\frac{c}{\alpha}-\frac{1080 \gamma}{11 \alpha} \operatorname{coth} \xi+\frac{120 \gamma}{\alpha} \operatorname{coth}^{3} \xi$

and the kink wave solution

$u_{18}(\xi)=\frac{c}{\alpha}-\frac{1080 \gamma}{11 \alpha} \tanh \xi+\frac{120 \gamma}{\alpha} \tanh ^{3} \xi$.

For $p=1, q=1$, we find $\beta=-76 \gamma / 11$ and thus gain the two periodic traveling wave solutions

$u_{19}(\xi)=\frac{c}{\alpha}-\frac{1080 \gamma}{11 \alpha} \tan \xi-\frac{120 \gamma}{\alpha} \tan ^{3} \xi$,

$u_{20}(\xi)=\frac{c}{\alpha}+\frac{1080 \gamma}{11 \alpha} \cot \xi+\frac{120 \gamma}{\alpha} \cot ^{3} \xi$.

For $p=1, q=-4$, we obtain $\beta=304 \gamma / 11$ and therefore determine the hyperbolic function solution

$u_{21}(\xi)=\frac{c}{\alpha}-\frac{1080 \gamma}{11 \alpha} \operatorname{coth}(2 \xi)+\frac{960 \gamma}{\alpha} \operatorname{coth}^{3}(2 \xi)$,

and the kink wave solution

$u_{22}(\xi)=\frac{c}{\alpha}-\frac{8640 \gamma}{11 \alpha} \tanh (2 \xi)+\frac{960 \gamma}{\alpha} \tanh ^{3}(2 \xi)$. 
For $p=1, q=4$, we find $\beta=-304 \gamma / 11$ and therefore obtain the two periodic traveling wave solutions

$$
\begin{aligned}
& u_{23}(\xi)=\frac{c}{\alpha}-\frac{8640 \gamma}{11 \alpha} \tan (2 \xi)-\frac{960 \gamma}{\alpha} \tan ^{3}(2 \xi), \\
& u_{24}(\xi)=\frac{c}{\alpha}+\frac{8640 \gamma}{11 \alpha} \cot (2 \xi)+\frac{960 \gamma}{\alpha} \cot ^{3}(2 \xi) .
\end{aligned}
$$

\section{Conclussions}

In this paper an auxiliary Riccati equation method is used to construct the exact solutions of the KuramotoSivashinsky equation with the aid of software Maple. As a result many kinds of exact traveling wave solutions are obtained, which show that this method is reliable and effective. The applied method will be used in further works to establish more entirely new solutions for other kinds of nonlinear wave equations. The availability of computer systems like Maple facilitates the complicated algebraic calculations, which allows us to solve tedious algebraic calculation.

\section{References}

[1] M. J. Ablowitz, P. A. Clarkson, “Solitons, nonlinear evolution equations and inverse scattering," London: Cambridge University Press, 1991.

[2] Hanze Liu, Jibin Li, Lei Liu, "Lie symmetry analysis, optimal systems and exact solutions to the fifthorder KdV types of equations,” Journal of Mathematical Analysis and Applications, vol. 368, pp. 551-558, 2010.

[3] Aiyong Chen, Jibin Li, "Single peak solitary wave solutions for the osmosis $\mathrm{K}(2,2)$ equation under inhomogeneous boundary condition,” Journal of Mathematical Analysis and Applications, vol. 369, pp.758-766, 2010.

[4] Dahe Feng, Jibin Li, “Exact explicit travelling wave solutions for the $(\mathrm{n}+1)$-dimensional field model," Physics Letters A, vol. 369, pp.255-261, 2007.

[5] A. M. Wazwaz, "A sine-cosine method for handling nonlinear wave equations,” Math.Comput. Model., vol. 40, pp. 499-508, 2004.

[6] Zhenya Yan, "New explicit travelling wave solutions for two new integrable coupled nonlinear evolution equations,” Physics Letters A, vol. 292, pp. 100-106, 2001.

[7] Mingliang Wang, "Exact solutions for a compound KdV-Burgers equation,” Physics Letters A, vol. 213, pp. 279-287, 1996.

[8] A. Wazwaz, "New solitary wave solutions to the Kuramoto-Sivashinsky and the Kawahara equations," Applied Mathematics and Computation, vol.182, pp. 1642-1650, 2006.

[9] J. Rademacher, R. Wattenberg, "Viscous shocks in the destabilized Kuramoto-Sivashinsky,” J. Comput. Nonlin. Dynam, vol. 1, pp. 336-347, 2006. 